TRANSACTIONS OF THE

AMERICAN MATHEMATICAL SOCIETY

Volume 348, Number 8, August 1996

\title{
WHICH FAMILIES OF $l$-MODAL MAPS ARE FULL?
}

\author{
R. GALEEVA AND S. VAN STRIEN
}

\begin{abstract}
In this paper we shall show that certain conditions which are sufficient for a family of one-dimensional maps to be full cannot be dispensed with.
\end{abstract}

We say that a continuous map $f:[0,1] \rightarrow[0,1]$ is $l$-modal if it has local extrema at $0=c_{0}<c_{1}<c_{2}<\cdots<c_{l}<c_{l+1}=1$ and if $f(\{0,1\}) \subset\{0,1\}$. Let $f_{\mu}:[0,1] \rightarrow[0,1], \mu \in \Delta$, be a $l$-modal family where $\Delta$ is some connected subset of a Euclidean space.

In this paper we want to discuss which conditions are necessary and sufficient for such a family to be full. Let us first give one definition of this notion. As usual we define the kneading invariant of $f$ as follows. Let $I_{i}=\left(c_{i}, c_{i+1}\right)$ and $\Sigma=\left\{I_{1}, c_{1}, I_{2}, c_{2}, \ldots, I_{l+1}\right\}^{\mathbb{N}}$ endowed with the metric $d(\underline{x}, y)=\sum_{i=0}^{\infty} \frac{1}{2^{i}} d\left(x_{i}, y_{i}\right)$ where $d\left(x_{i}, y_{i}\right)=1$ if $x_{i} \neq y_{i}$ and $d\left(x_{i}, x_{i}\right)=0$. Let $\underline{i}:[0,1] \rightarrow \Sigma$ be defined by $\underline{i}(x)=\left(i_{0}(x), i_{1}(x), \ldots, i_{n}(x), \ldots\right)$ where $i_{n}(x)=I_{k}$ if $f^{n}(x) \in I_{k}$ and $i_{n}(x)=c_{k}$ if $f^{n}(x)=c_{k}$. The sequence $\underline{i}(x)$ is called the itinerary of $x$ under $f$. Given $n \in \mathbb{N}$ and $x \in I$, there exists $\delta>0$ such that $i_{n}(y)$ is constant on the interval $(x, x+\delta)$. It follows that the one-sided limit $\underline{i}\left(x^{+}\right)=\lim _{y \downarrow x} \underline{i}(y)$ always exists. The sequences $\nu_{1}, \ldots, \nu_{l}$ defined by

$$
\nu_{i}=\underline{i}\left(c_{i}^{+}\right)
$$

are called the kneading invariants of $f$.

For the purposes of this paper (see the remark below the next theorem), we say that a $l$-modal family $f_{\mu}:[0,1] \rightarrow[0,1], \mu \in \Delta$, is full if the following property holds. Given a $l$-modal map $g:[0,1] \rightarrow[0,1]$ such that

1. no lap of $g$ is a homterval (an interval $J$ is called a homterval for $g$ if $g^{n} \mid J$ is a homeomorphism for each $n$ );

2. $g(0)=f_{\mu}(0)$ and $g(1)=f_{\mu}(1)$ for $\mu \in \Delta$,

there exists $\mu^{\prime} \in \Delta$ such that $g$ and $f_{\mu^{\prime}}$ have the same kneading invariants.

Let us first cite a theorem which gives a sufficient condition for a family to be full. In order to state this theorem we need to introduce the map $F: \Delta \rightarrow[0,1]^{l}$ defined by

$$
F(\mu)=\left(f_{\mu}\left(c_{1}\right), \ldots, f_{\mu}\left(c_{l}\right)\right) .
$$

Since successive turning points of $f_{\mu}$ are alternating local maxima and local minima, the map $F$ has values in

$$
V=\left\{\left(v_{1}, \ldots, v_{l}\right) \in I^{l} ; s(-1)^{i}\left(v_{i+1}-v_{i}\right)<0 \text { for } i=0, \ldots, l\right\}
$$

Received by the editors June 6, 1994 and, in revised form, September 21, 1995.

1991 Mathematics Subject Classification. Primary 58Fxx, 34Cxx, 30-xx.

Key words and phrases. One-dimensional dynamics. 
where $s \in\{-,+\}$ depending on the orientation of $f_{\mu}$ and where $v_{0}$ and $v_{l+1}$ are respectively the images of the left and right endpoint of $I$. (By the definition of an $l$-modal map, $v_{0}, v_{l+1} \in\{0,1\}$.) Here, and below, we have written $f_{\mu}\left(c_{i}\right)$ instead of $f_{\mu}\left(c_{i}(\mu)\right)$.

In the unimodal case, there is a well-known condition which suffices for a family to be full: for this it is sufficient that the map

$$
\Delta \ni \mu \mapsto F(\mu) \in \Delta=[0,1] \backslash\{F(0)=F(1)\}
$$

is surjective, see [M.T.] and [C.E.]. In [M.S.] this result is generalized:

Theorem 1 (See [M.S.]). Assume that $f_{\mu}, \mu \in \Delta$, is a family of l-modal $C^{1}$ maps such that

1. $\Delta \ni \mu \mapsto f_{\mu} \in C^{1}$ extends continuously to a map $\bar{\Delta} \ni \mu \mapsto f_{\mu} \in C^{1}$ and that the turning points $c_{1}(\mu), c_{2}(\mu), \ldots, c_{l}(\mu)$ depend continuously on $\mu \in \Delta$;

2. $F: \Delta \rightarrow V$ is a homeomorphism

then $f_{\mu}$ is a full family.

Proof. In the unimodal case one can use the order structure on the space of kneadings. In the general case one can proceed as in [M.S.].

The purpose of this paper is to show that neither of the two conditions in the above theorem can be dropped.

Remark 1. Let us discuss the notion of full family in a little more detail. For this notion to be useful one might hope that polynomial families are full. However, maps from such families do not have wandering intervals, see [M.S.]. So we would like to make sure that even if $g$ has homtervals, the fact that $f_{\mu}$ and $g$ have the same kneading invariants does not imply that $f_{\mu}$ also has wandering intervals. So let us check that we have defined everything so that we indeed do not force wandering intervals! Let us define $x \sim y$ if the interval connecting $x$ and $y$ is a union of homtervals of $g$. Note that $g / \sim:[0,1] / \sim \rightarrow[0,1] / \sim$ is a continuous map (since the image of a homterval is again a homterval). Because of condition 1 above, $g / \sim$ has the same modality as $g$. (In fact, if we did not impose condition 1 then $g$ could be of the form $g(x)=x$ for $x \in[0,1 / 2]$ and $g(x)=1-x$ for $x \in[1 / 2,1]$ in which case $[0,1] / \sim$ would be a single point.) Clearly, the kneading invariants of $g$ and $g / \sim$ are the same: $\nu_{i}(g)=\nu_{i}(g / \sim)$. (This is the reason to choose the kneading invariants $\nu_{i}$ from above, rather than the alternative kneading invariants $\hat{\nu}_{i}(g)=\underline{i}\left(g\left(c_{i}\right)\right)$, because if the interval connecting $g^{n}\left(c_{i}\right)$ and $c_{j}$ is a homterval then $\hat{\nu}_{i}(g) \neq \hat{\nu}_{i}(g / \sim)$. So we cannot take the quotient $g / \sim$ of $g$ without changing these alternative kneading invariants.)

Remark 2. In [M.S.], maps were defined to be essentially combinatorially equivalent if 'the dynamics inside periodic attractors' also matched. If two such maps are equivalent then they certainly have the same kneading invariants. In [M.S.], a family $f_{\mu}$ was called full, if for any $g$ as above - but satisfying some additional hypothesis - there exists $\mu$ such that $f_{\mu}$ and $g$ are essentially combinatorially equivalent. The additional conditions on $g$ in the theorem in [M.S.] become irrelevant if we only care about the kneading invariant of $g$. This is the reason why Theorem 1 follows from Theorem II.4.1 from [M.S.].

Remark 3. As is shown in [M.S.], the two assumptions in the statement of Theorem 1 can be weakened. 
In Theorem 2 we shall show that condition 1 in Theorem 1 is necessary. That is, we shall show that if for each $\mu$ the map $f_{\mu}$ has a non-zero derivative in one of its turning points then $f_{\mu}$ cannot be a full family. Since $\Delta$ is not compact, we shall need to assume in Theorem 2 that $f_{\mu}$ does not degenerate too much as $\mu \rightarrow \partial \Delta$. To be specific, we shall assume in Theorem 2 that there exists $K<\infty$ such that for the family of $\ell$-modal maps $f_{\mu}$ one has

1. for each $i=1,2, \ldots, \ell+1$ the restriction $f_{\mu} \mid I_{i}(\mu)$ is $C^{2}$ and $\left|D^{2} f_{\mu}(x)\right| \leq K$ for each $\mu \in \Delta$ and each $x \in I_{i}(\mu)=\left(c_{i}(\mu), c_{i+1}(\mu)\right)$;

2. small derivatives only occur at the boundary of intervals of monotonicity or when the image of the whole interval is small: for each $\epsilon>0$ there exists $\delta>0$ such that $\left|D f_{\mu}(x)\right| \leq \delta$ for some $\mu \in \Delta$ and $x \in I_{i}(\mu)$ then either $d\left(x_{n},\left\{c_{1}, \ldots, c_{l}\right\}\right) \leq \epsilon$ or $\left|f_{\mu}\left(I_{i}(\mu)\right)\right| \leq \epsilon$ (here $|U|$ stands for the length of an interval $U$ );

3. the intervals of monotonicity do not shrink to zero: $\left|c_{i}(\mu)-c_{i+1}(\mu)\right| \geq 1 / K$ for all $\mu \in \Delta$ and each $i=0,1,2, \ldots, \ell$;

4. one of the following two conditions is satisfied:

4(a) if 0 (resp. 1) is a fixed point then for each sequence of points $x_{n}$ and parameters $\mu_{n}$ such that $x_{n}, f_{\mu_{n}}\left(x_{n}\right), \ldots, f_{\mu_{n}}^{n}\left(x_{n}\right) \in I_{1}$ (resp. $\left.\in I_{l+1}\right)$ for which $x_{n}$ is not in the basin of an attracting fixed point of $f_{\mu_{n}}$ we have $x_{n} \rightarrow 0$ (resp. $x_{n} \rightarrow 1$ );

4 (b) if 0 and 1 are both not fixed points then the endpoints are exchanged by $f$ (i.e., $\left.f_{\mu}(0)=1, f_{\mu}(1)=0\right)$ and then we assume that for each sequence of points $x_{n}$ and parameters $\mu_{n}$ such that $x_{n}, f_{\mu_{n}}^{2}\left(x_{n}\right), \ldots, f_{\mu_{n}}^{2 n}\left(x_{n}\right) \in I_{1}$ for which $x_{n}$ is not in the basin of an attracting point of period 2 of $f_{\mu_{n}}$ we have $x_{n} \rightarrow 0$.

The last assumption prohibits that there exists a sequence of maps in the family $f_{\mu}$ which more and more 'almost' have a fixed point. This would happen, for instance, if a limit map has a saddle-node fixed point. If, for example, the left endpoint of the interval is a fixed point, then it is prohibited that an orbit remains longer and longer in $I_{1}$ without converging to an attracting fixed point in $I_{1}$. For all reasonable families of maps this condition is satisfied because of some type of convexity. Certainly families of piecewise affine maps satisfy the above assumptions.

Theorem 2 (Condition 1 from Theorem 1 is necessary). Suppose that $f_{\mu}$ is as above and satisfies the conditions from Theorem 1, except that $f_{\mu}$ is not differentiable in at least one of the turning points $c_{i}(\mu)$ in the sense that there exists $k^{\prime}>0$ and $\delta>0$ such that $\left|D f_{\mu}(x)\right| \geq k^{\prime}>0$ for all $\mu \in \Delta$ and all $x \in\left(c_{i}(\mu)-\delta, c_{i}(\mu)+\delta\right)$ with $x \neq c_{i}(\mu)$. Then $f_{\mu}$ is not full.

We should emphasize that the above theorem applies to any family of piecewise linear maps or piecewise polynomial maps where the slope at one of the critical points is bounded away from zero. Such families are therefore not full.

Proof. In order to be definite, assume that $f_{\mu}(0)=f_{\mu}(1)=0$ and that $c_{i}$ is a local maximum. The other case can be dealt with similarly. To show that $f_{\mu}$ is not a full family we choose a sequence of smooth $\ell$-modal maps $g_{n}:[0,1] \rightarrow[0,1]$ and show that for sufficiently large $n$, there exists no $\mu \in \Delta$ for which $f_{\mu}$ and $g_{n}$ have the same kneading sequence. So choose smooth $\ell$-modal maps $g_{n}$ with $g_{n}(0)=g_{n}(1)=0$ with the following properties. Take $g_{n}$ so that $g_{n}\left(c_{k}\right)$ is equal to 0 or 1 except when 
$k=i$. So the image of each lap, except that of $I_{i-1}$ and $I_{i}$, is equal to the entire interval $[0,1]$. Furthermore, we choose $g_{n}\left(c_{i}\right) \in I_{l+1}$ so that it is 'close' to 1 in the following precise fashion:

i) $g_{n}^{2}\left(c_{i}\right), \ldots, g_{n}^{n+1}\left(c_{i}\right) \in I_{1}$,

ii) $g_{n}^{n+2}\left(c_{i}\right) \in I_{i-1}=\left(c_{i-1}, c_{i}\right)$,

iii) $g_{n}^{n+3}\left(c_{i}\right) \in I_{l+1}$,

iv) $g_{n}^{n+4}\left(c_{i}\right), \ldots, g_{n}^{2 n+3}\left(c_{i}\right) \in I_{1}$, and

v) $g_{n}^{2 n+4}\left(c_{i}\right) \in I_{i}=\left(c_{i}, c_{i+1}\right)$ is a fixed point of the map $g_{n}^{n+2}$.

Clearly $g_{n}$ can be chosen so that i)-iv) hold. Let us explain why we can choose $g_{n}$ so that property v) also holds. Since $g_{n} \mid I_{i-1}$ is increasing ii) implies that $g_{n}^{n+3}\left(c_{i}\right)$ lies to the left of $g_{n}\left(c_{i}\right)$ in $I_{l+1}$. Since $g_{n} \mid I_{l+1}$ is decreasing this implies that $g_{n}^{n+4}\left(c_{i}\right)$ lies to the right of $g_{n}^{2}\left(c_{i}\right)$ in $I_{1}$. Hence $g_{n}^{n+4+j}$ lies to the right of $g_{n}^{2+j}$ for $j=0,1, \ldots, n$. This means that we can modify $g_{n}$ so that $g_{n}^{2 n+4}\left(c_{i}\right)$ is an arbitrary point in $I_{i}$. In particular, taking $p \in I_{i}$ so that $g_{n}(p)=g_{n}\left(g_{n}^{n+2}\left(c_{i}\right)\right)$, we can make sure that $g_{n}^{2 n+4}\left(c_{i}\right)=p$. This implies that $p$ is a fixed point of $g_{n}^{n+2}$. In terms of kneading sequences, i)-v) read as

$$
\underline{i}\left(c_{i}\right)=c_{i} I_{l+1} I_{1}^{n} I_{i-1}\left(I_{l+1} I_{1}^{n} I_{i}\right)^{\infty} .
$$

The above choice implies that the restriction of $g_{n}^{n+2}$ to some interval containing the point $c_{i}$ is a full unimodal map. More precisely, $T_{n}=\left[g_{n}^{n+2}\left(c_{i}\right), g_{n}^{2 n+4}\left(c_{i}\right)\right]$ contains $c_{i}$, the intervals $T_{n}, \ldots, g_{n}^{n+1}\left(T_{n}\right)$ are disjoint and $g_{n}^{n+2}$ is a unimodal map from $T_{n}$ onto $T_{n}$. Moreover, $g_{n}\left(T_{n}\right) \subset I_{l+1}$ and $g_{n}^{i}\left(T_{n}\right) \subset I_{1}$ for $i=1,2, \ldots, n+1$. (In fact, this also implies that $g_{n}^{n+2} \mid T$ has a local minimum at $c_{i}$ because $g_{n}$ has a local maximum at $c_{i}, g \mid I_{l+1}$ is orientation reversing and $g \mid I_{1}$ is orientation preserving.) This choice implies that no lap of $g_{n}$ is a homterval and so the $\ell$-modal map $g_{n}$ satisfies all the required conditions.

Let us assume by contradiction that for each $n$ there exists $\mu_{n} \in \Delta$ for which $f_{\mu_{n}}$ and $g_{n}$ have the same kneading invariants. First notice that the turning points of $g_{n}$ are eventually periodic. Moreover, by construction of $g_{n}$ no two distinct iterates of turning points are in a homterval. It follows that the turning points of $f_{\mu_{n}}$ are also eventually periodic and that if we take $U_{n}=\left[f_{\mu_{n}}^{n+2}\left(c_{i}\right), f_{\mu_{n}}^{2 n+4}\left(c_{i}\right)\right]$ then $U_{n} \ni c_{i}$ and

- $U_{n}, \ldots, f_{\mu_{n}}^{n+1}\left(U_{n}\right)$ are disjoint;

- $f_{\mu_{n}}\left(U_{n}\right) \subset I_{l+1}$

- $f_{\mu_{n}}^{2}\left(U_{n}\right), \ldots, f_{\mu_{n}}^{n+1}\left(U_{n}\right) \subset I_{1}$

- $f_{\mu_{n}}^{n+2}$ is a unimodal map from $U_{n}$ onto $U_{n}$;

- $f_{\mu_{n}}\left(I_{j}\right)=[0,1]$ except for $j=i-1, i$.

First notice that $\left|f_{\mu_{n}}\left(U_{n}\right)\right|$ is at least $\left(k^{\prime} / 2\right)\left|U_{n}\right|$ for $n$ sufficiently large, because of the assumption on $D f_{\mu_{n}}$ near $c_{i}$. Moreover, because of assumptions 2 and 3 , there exists a uniform constant $C>0$ such that $\left|f_{\mu_{n}}^{2}\left(U_{n}\right)\right| \geq C\left|U_{n}\right|$. Now let $a_{n}$ be the left endpoint of $U_{n}^{\prime}=f_{\mu_{n}}^{2}\left(U_{n}\right)$. Then $U_{n}^{\prime}, \ldots, f_{\mu(n)}^{n-1}\left(U_{n}^{\prime}\right) \subset I_{1}, f_{\mu_{n}}^{n}\left(U_{n}^{\prime}\right) \ni c_{i}$ are disjoint (and lie in increasing order). Let $W_{n}$ be the 'fundamental neighbourhood' $W_{n}=$ $\left[a_{n}, f\left(a_{n}\right)\right]$ (it strictly contains $\left.U_{n}^{\prime}\right)$. Since $W_{n}, \ldots, f_{\mu_{n}}^{n-2}\left(W_{n}\right)$ are all contained in $I_{1}$, assumption 4 implies that $W_{n} \rightarrow 0$. Because of assumption 3 we get that $\left|f_{\mu_{n}}^{n-2}\left(W_{n}\right)\right|$, and therefore $\left|f_{\mu_{n}}^{n}\left(W_{n}\right)\right|$ is bounded away from zero. Hence

$$
\left|f_{\mu_{n}}^{n}\left(W_{n}\right)\right| /\left|W_{n}\right| \rightarrow \infty \text { as } n \rightarrow \infty
$$


Notice that $x \mapsto \log \left|D f_{\mu_{n}}(x)\right|$ is uniformly Lipschitz on the interval $J_{\mu_{n}}$ connecting 0 to $I_{1} \cap f_{\mu_{n}}^{-1}\left(c_{i}\right)$ (here we use that this last point is not too close to $c_{1}$ and assumption 2. Therefore

$$
\begin{gathered}
\log \frac{\left|D f_{\mu_{n}}^{n}(u)\right|}{\left|D f_{\mu_{n}}^{n}(v)\right|}=\sum_{i=0}^{n-1} \log \left|D f_{\mu_{n}}\left(f_{\mu_{n}}^{i}(u)\right)\right|-\log \left|D f_{\mu_{n}}\left(f_{\mu_{n}}^{i}(v)\right)\right| \\
\leq K \sum_{i=0}^{n}\left|f_{\mu_{n}}^{i}(u)-f_{\mu_{n}}^{i}(v)\right| \leq K
\end{gathered}
$$

for $u, v \in W_{n}$. Combining this with (1) we get that there exists a function $C(n)$ with $C(n) \rightarrow \infty$ as $n \rightarrow \infty$ such that

$$
\frac{\left|f_{\mu_{n}}^{n}\left(U_{n}^{\prime}\right)\right|}{\left|U_{n}^{\prime}\right|} \geq C(n)
$$

In particular for large $n$, there exists no $\mu_{n} \in \Delta$ for which $f_{\mu_{n}}$ maps some interval $U_{n}$ in $n+2$ steps into itself (in the required way).

Q.E.D.

Naively, one might think that condition 2 in Theorem 1 can be replaced by the condition that $F$ is surjective. This is false!

Theorem 3 (Condition 2 in Theorem 1 is necessary). There exists a family $f_{\mu}:[0,1] \rightarrow[0,1]$ of bimodal maps which satisfies condition 1 from Theorem 1, for which $F: \Delta \rightarrow V$ is surjective, but which is not full.

Proof. We will give the proof of Theorem 3 in two steps. The idea of the construction is to take a cubic family $P_{\mu}$ which is full, remove a point $\mu_{0}$ from the parameter space $\Delta$, excluding in this way some type of combinatorial behaviour (kneading invariant). As a consequence the family $P_{\mu}, \mu \in \Delta \backslash\left\{\mu_{0}\right\}$ is nonfull. Next we choose a parameter-dependent coordinate change $h_{\mu}$, so that the extremal value map $\hat{F}: \Delta \rightarrow V$ corresponding to $h_{\mu} \circ P_{\mu} \circ h_{\mu}^{-1}, \mu \in \Delta \backslash\left\{\mu_{0}\right\}$ becomes surjective again.

Step 1 . Let us consider the cubic family:

$$
P_{\mu}(x)=(1-a-b) x^{3}+b x^{2}+a x
$$

where

$$
\mu=(a, b) \in \Delta=\left\{\mu=(a, b) ; P_{\mu}:[0,1] \rightarrow[0,1] \text { is bimodal }\right\} .
$$

Let $V$ be the set of extremal values, i.e., let $v_{1}=P_{\mu}\left(c_{1}\right)$ and $v_{2}=P_{\mu}\left(c_{2}\right)$ and define

$$
V=\left\{\left(v_{1}, v_{2}\right) ; 1 \geq v_{1}>v_{2} \geq 0\right\} .
$$

According to the Corollary to Theorem II.4.1 in [M.S.], the associated map $F: \Delta \rightarrow$ $V$ is a homeomorphism. In particular, it is a full family and therefore there exists a parameter $\mu_{0}=\left(a_{0}, b_{0}\right) \in \Delta$ for which $P_{\mu_{0}}\left(c_{1}\right)=c_{2}, P_{\mu_{0}}\left(c_{2}\right)=c_{1}$, i.e., $P_{\mu_{0}}$ has a superstable cycle, containing both turning points. Since there exists no parameter $\mu \neq \mu_{0}$ such that $P_{\mu}$ and $P_{\mu_{0}}$ have the same kneading invariants (see [M.T.] or Section II.10 in [M.S.]), the family $P_{\mu}(x), \mu \in \Delta \backslash\left\{\mu_{0}\right\}$ is not full and also the map $F: \Delta \backslash\left\{\mu_{0}\right\} \rightarrow V$ is not surjective.

Step 2. Now we will choose a parameter dependent diffeomorphism $h_{\mu}(x)$ such that

$$
\Delta \backslash\left\{\mu_{0}\right\} \ni \mu \mapsto \hat{F}(\mu) \in V
$$


is surjective. Here $\hat{F}$ is the map which associates to $\mu$ the extremal values of $h_{\mu} \circ f_{\mu} \circ h_{\mu}^{-1}$. This means that

$$
\hat{F}(\mu)=\left(h_{\mu}\left(f_{\mu}\left(c_{1}(\mu)\right)\right), h_{\mu}\left(f_{\mu}\left(c_{2}(\mu)\right)\right)\right) .
$$

To construct $h_{\mu}$ we fix $\epsilon_{0}>0$ so small that the $2 \epsilon_{0}$ neighbourhoods of $c_{i}\left(\mu_{0}\right)$ are contained in $(0,1)$ and are disjoint. Moreover let $p=\left(c_{1}\left(\mu_{0}\right)+c_{2}\left(\mu_{0}\right)\right) / 2$ and define two smooth functions $\rho_{i}: \mathbb{R} \rightarrow[0,1]$ such that $\operatorname{supp}\left(\rho_{1}\right) \subset[0, p]$ and $\operatorname{supp}\left(\rho_{2}\right) \subset[p, 1]$ and such that $\rho_{i}=1$ for $x \in\left[c_{i}-\epsilon_{0}, c_{i}+\epsilon_{0}\right]$. Define $A=\sup _{x \in[0,1]}\left\{\left|D \rho_{1}(x)\right|,\left|D \rho_{2}(x)\right|\right\}$ and choose $\epsilon \in\left(0, \epsilon_{0}\right)$ so that $\epsilon A<1$ and $B_{\epsilon}\left(v_{1}\left(\mu_{0}\right), v_{2}\left(\mu_{0}\right)\right) \subset V$ (here $B_{t}(z)$ is the ball with radius $t$ centered in $z \in \mathbb{R}^{2}$ ). Choose $\delta>0$ so small that $F\left(B_{\delta}\left(\mu_{0}\right)\right) \subset B_{\epsilon / 2}\left(v_{1}\left(\mu_{0}\right), v_{2}\left(\mu_{0}\right)\right)$. Next we consider a surjective smooth function $\theta: \mathbb{R} \rightarrow[-\epsilon,+\epsilon]$ so that $\operatorname{supp}(\theta) \subset\left(0, \delta^{2}\right]$. Define $\Psi: \Delta \backslash\left\{\mu_{0}\right\} \rightarrow \mathbb{R}^{2}$ by

$$
\Psi(\mu)=\left(\Psi_{1}(\mu), \Psi_{2}(\mu)\right):=\theta\left(\left\|\mu-\mu_{0}\right\|^{2}\right) \cdot \frac{\left(\mu-\mu_{0}\right)}{\left\|m u-\mu_{0}\right\|} .
$$

Since $\operatorname{supp}(\theta) \subset\left(0, \delta^{2}\right]$, the map $\Psi$ is smooth. Furthermore, define

$$
h_{\mu}(x)=x+\Psi_{1}(\mu) \rho_{1}(x)+\Psi_{2}(\mu) \rho_{2}(x) .
$$

Since $\operatorname{supp}\left(\rho_{1}\right)$ and $\operatorname{supp}\left(\rho_{2}\right)$ are disjoint sets and since $\left|\Psi_{i}\right|\left|D \rho_{i}\right| \leq \epsilon A<1$, it follows that $D h_{\mu}(x)>0$ for $x \in[0,1]$ and that $[0,1] \ni x \mapsto h_{\mu}(x) \in[0,1]$ is a diffeomorphism. Moreover, $(\mu, x) \mapsto h_{\mu}(x)$ is smooth.

Next we show that $\hat{F}$ is surjective. By the choice of $\rho_{i}$,

$$
h_{\mu}\left(f_{\mu}\left(c_{i}(\mu)\right)\right)=f_{\mu}\left(c_{i}(\mu)\right)+\Psi_{i}(\mu)
$$

and therefore

$$
\hat{F}(\mu)=F(\mu)+\Psi(\mu) .
$$

Because $\operatorname{supp}(\theta) \subset\left[0, \delta^{2}\right]$ we have that $\Psi(\mu)=0$ for $\mu \notin B_{\delta}\left(\mu_{0}\right)$. Hence

$$
\hat{F}\left(\Delta \backslash B_{\delta}\left(\mu_{0}\right)\right)=F\left(\Delta \backslash B_{\delta}\left(\mu_{0}\right)\right) \supset V \backslash B_{\epsilon}\left(v_{1}\left(\mu_{0}\right), v_{2}\left(\mu_{0}\right)\right) .
$$

Since $\theta$ is surjective there exists $\delta_{1}, \delta_{2} \in(0, \delta)$ such that $\theta\left(\delta_{1}^{2}\right)=-\epsilon$ and $\theta\left(\delta_{2}^{2}\right)=\epsilon$. Hence

- $\Psi\left(\partial B_{r}\left(\mu_{0}\right)\right)=\partial B_{\epsilon}(0)$ for $r=\delta_{i}$;

- the winding number of the parameterized closed loop

$$
S^{1} \ni \phi \mapsto \Psi\left(\mu_{0}+(r \cos \phi, r \sin \phi)\right)
$$

with respect to a point $z$ in $B_{\epsilon}(0)$ depends on $r \in[0, \delta]$ : it is equal to $(-1)^{i}$ if $r=\delta_{i}$. (In fact, for some $r$ it is not defined because the loop goes through z.)

From $\hat{F}(\mu)=F(\mu)+\Psi(\mu)$, since $\Psi\left(\partial B_{r}\left(\mu_{0}\right)\right)=\partial B_{\epsilon}(0)$ for $r=\delta_{i}$ and because $F\left(B_{\delta}\left(\mu_{0}\right)\right) \subset B_{\epsilon / 2}\left(v_{1}\left(\mu_{0}\right), v_{2}\left(\mu_{0}\right)\right)$ it follows that for $r=\delta_{i}$ the loop

$$
\gamma: \quad S^{1} \ni \phi \mapsto \hat{F}\left(\mu_{0}+(r \cos \phi, r \sin \phi)\right)
$$

lies outside $B_{\epsilon / 2}\left(v_{1}\left(\mu_{0}\right), v_{2}\left(\mu_{0}\right)\right)$ and that its winding number w.r.t. a point $z$ in the disc $B_{\epsilon / 2}\left(v_{1}\left(\mu_{0}\right), v_{2}\left(\mu_{0}\right)\right)$ is still equal to $(-1)^{i}$ when $r=\delta_{i}$. Hence

$$
\hat{F}\left(B_{\delta}\left(\mu_{0}\right) \backslash\left\{\mu_{0}\right\}\right) \supset B_{\epsilon / 2}\left(v_{1}\left(\mu_{0}\right), v_{2}\left(\mu_{0}\right)\right),
$$


because otherwise there exists a point $z \in B_{\epsilon / 2}\left(v_{1}\left(\mu_{0}\right), v_{2}\left(\mu_{0}\right)\right)$ such that $z \notin$ the loop $\gamma$ for each $r \in[0, \delta]$. This would imply that the winding number of $\gamma$ with respect to $z$ would be independent of $r$, which contradicts our former assertion. It follows that $\hat{F}: \Delta \backslash\left\{\mu_{0}\right\} \rightarrow V$ is surjective even though $P_{\mu}, \mu \in \Delta \backslash\left\{\mu_{0}\right\}$, is not a full family.

Q.E.D.

\section{ACKNOWLEDGEMENT}

We would like to thank the referee for his very useful and careful comments.

Addition: After this paper was submitted we learned about related preprints by Jimenez López and Snoha, There are no Piecewise Linear Maps of type $2^{\infty}$, and by Martens and Tresser, Forcing of periodic orbits and renormalization of piecewise affine maps. From these papers it follows that piecewise affine maps are never infinitely renormalizable. The maps from our families in Theorem 1 are more general, but as a consequence we can only show that these families cannot contain infinite "kneadings" of arbitrary maps (and thus show that the families cannot be full).

\section{REFERENCES}

[C.E.] P. Collet and J.-P. Eckmann: Iterated Maps of the Interval as Dynamical Systems. Birkhäuser, Boston (1980). MR 82j:58078

[M.T.] J. Milnor and W. Thurston: On iterated maps of the interval: I,II. In: "Dynamical Systems: Proc. Univ, of Maryland 1986-87", Lecture Notes in Mathematics 1342, (1988), 465-563. MR 90a:98083

[M.S.] W. de Melo and S. van Strien, One-dimensional dynamics. Ergebnisse Series 25, Springer Verlag, Berlin (1993). MR 95a:58035

Department of Mathematics, Northwestern University, Evanston, Illinois 60201

Current address: UMR 129 CNRS UNSA, 1361 Route des Lucioles Sophia Antipolis, 06560 Valbonne, France

E-mail address: galeeva@doublon.unice.fr

Department of Mathematics, University of Amsterdam, Amsterdam, The NetherLANDS

E-mail address: strien@fwi.uva.nl 